\title{
Water hammer with fluid-structure interaction in thick-walled pipes
}

\author{
A.S. Tijsseling \\ Department of Mathematics and Computer Science, Eindhoven University of Technology, P.O. Box 513, \\ 5600 MB Eindhoven, The Netherlands
}

Tel: +31402472755

Fax: +31402442489

E-mail address: a.s.tijsseling@tue.nl

\begin{abstract}
A one-dimensional mathematical model is presented which describes the acoustic behaviour of thickwalled liquid-filled pipes. The model is based on conventional water-hammer and beam theories. Fluidstructure interaction (FSI) is taken into account. The equations governing straight pipes are derived by the cross-sectional integration of axisymmetric two-dimensional basic equations. The resulting FSI four-equation model has small correction terms and factors accounting for the wall thickness. Exact solutions of this model show that these corrections are important only for very thick pipes, with, say, a radius/thickness ratio smaller than 2.
\end{abstract}

Keywords: Water hammer; Fluid transients; Fluid-structure interaction (FSI); Pipe flow 


\section{Introduction}

Water hammer concerns the generation, propagation and reflection of pressure waves in liquid-filled pipe systems. These waves may have very steep fronts when generated by, for example, the closure of lightly damped check valves [1] or the collapse of liquid column separations [2]. Steep-fronted waves are prone to excite the structural system and as a result make individual pipes move. Pipe motion itself generates water hammer, thus invoking fluid-structure interaction.

The numerical simulation of water-hammer events (with and without fluid-structure interaction) is usually based on one-dimensional mathematical models assuming thin-walled cylindrical pipes [3]. The ratio $e / R$ of wall-thickness to pipe-radius is then small with respect to unity, circumferential (hoop) stresses are uniform in the wall cross-section, and radial stresses are neglected. The present paper considers water hammer with axial/radial vibration of thick-walled pipes. The distributions of hoop and radial stresses in the wall are given as quasi-static functions of the internal and external pressures. Cross-sectional averages are taken to arrive at a convenient one-dimensional formulation. The motivation for the research lies in the fact that many pipes in practice have relatively thick walls, for example, high-pressure pipes in chemical and nuclear industries, ductile-iron pipes with concrete lining, and plastic pipes.

The governing equations can be solved exactly in the time and frequency domains. The influence of wall thickness is studied from figures and tables showing wave propagation speeds, natural frequencies and pressure histories. Predictions from thin- and thick-walled theories are compared with experimental data obtained in a relatively thick pipe.

\section{Governing equations for the liquid}

Liquid pipe-flow is described by the following two-dimensional equations [4]:

Continuity equation (2D)

$$
\frac{1}{K} \frac{\partial p}{\partial t}+\frac{\partial v_{z}}{\partial z}+\frac{1}{r} \frac{\partial}{\partial r}\left(r v_{r}\right)=0
$$


Equation of motion in axial direction (2D)

$$
\rho_{f} \frac{\partial v_{z}}{\partial t}+\frac{\partial p}{\partial z}=0
$$

Equation of motion in radial direction (2D)

$$
\rho_{f} \frac{\partial v_{r}}{\partial t}+\frac{\partial p}{\partial r}=0
$$

The pressure $p$, the axial velocity $v_{z}$ and the radial velocity $v_{r}$ are functions of time $t$ and of the cylindrical coordinates $z$ (axial) and $r$ (radial). The circumferential co-ordinate $(\varphi)$ is omitted because of axial symmetry. Although the liquid density $\rho_{f}$ is taken constant, the liquid compressibility (elasticity) is present in the first term of the continuity equation (1) in which $K$ is the bulk modulus of the liquid. Gravity, viscosity and convective terms $\left[v_{z} \ll c_{f}\right.$, Eq. (43a), $\left.\mathrm{d} z / \mathrm{d} t=c_{f}\right]$ are ignored herein, so that a linear model is obtained. For an extended nonlinear model the reader is referred to [5].

To arrive at a one-dimensional formulation, in accordance with classical water-hammer theory, the equations (1) and (2) are multiplied by $2 \pi r$, integrated with respect to $r$ from 0 to $R$, the inner radius of the pipe, and divided by $A_{f}=\pi R^{2}$. The result is:

Continuity equation (1D)

$$
\frac{1}{K} \frac{\partial P}{\partial t}+\frac{\partial V}{\partial z}+\frac{2}{R} v_{r} I_{r=R}=0
$$

Equation of motion in axial direction (1D)

$$
\rho_{f} \frac{\partial V}{\partial t}+\frac{\partial P}{\partial z}=0
$$

in which

$$
V=\frac{1}{\pi R^{2}} \int_{0}^{R} 2 \pi r v_{z} \mathrm{~d} r \quad \text { and } \quad P=\frac{1}{\pi R^{2}} \int_{0}^{R} 2 \pi r p \mathrm{~d} r
$$

are the cross-sectionally averaged axial velocity and pressure, respectively. The first integral in Eqs (6) is the flow rate (discharge), the second the axial force due to pressure.

The radial equation of motion (3) is multiplied by $2 \pi r^{2}$, integrated with respect to $r$ from 0 to $R$, and divided by $2 \pi R^{2}$. The result is: 
Equation of motion in radial direction (1D)

$$
\left.\frac{1}{2} \rho_{f} R \frac{\partial v_{r}}{\partial t}\right|_{r=R}+\left.p\right|_{r=R}-P=0
$$

In deriving this equation it has been assumed that $r v_{r}=\left.R v_{r}\right|_{r=R}$, which is in accordance with the continuity equation (1) if compressibility is disregarded $(K=\infty)$ and axial inflow $\left(v_{z}\right)$ is concentrated at the central axis by means of a point source (singularity). Walker and Phillips [6] found the same equation by using asymptotic expansions. If one assumes that $R v_{r}=\left.r v_{r}\right|_{r=R}$, which corresponds to a linear distribution of the radial liquid velocity, the factor $\frac{1}{2}$ (representing the total liquid mass) becomes $\frac{1}{4}$ [Ref. 7] or $\frac{1}{6}$ [Ref. 8].

\section{Governing equations for the pipe}

Pipe motion is described by the following two-dimensional equations [4]; see Figs 1 and 2 for definition sketches:

Equation of motion in axial direction (2D)

$$
\rho_{t} \frac{\partial \dot{u}_{z}}{\partial t}=\frac{\partial \sigma_{z}}{\partial z}
$$

Equation of motion in radial direction (2D)

$$
\rho_{t} \frac{\partial \dot{u}_{r}}{\partial t}=\frac{1}{r} \frac{\partial\left(r \sigma_{r}\right)}{\partial r}-\frac{\sigma_{\varphi}}{r}
$$

with $\dot{u}_{z}$ and $\dot{u}_{r}$ the axial and radial pipe velocities, $\sigma_{z}$ and $\sigma_{r}$ the axial and radial stresses, $\sigma_{\varphi}$ the hoop stress, and $\rho_{t}$ the mass density of the wall material. The thickness of the pipe wall is denoted by $e$. Gravity, convective terms $\left[\dot{u}_{z} \ll c_{t}\right.$, Eq. (43b), $\left.\mathrm{d} z / \mathrm{d} t=c_{t}\right]$, shear forces, bending stiffness, rotatory inertia and structural damping are neglected herein. 


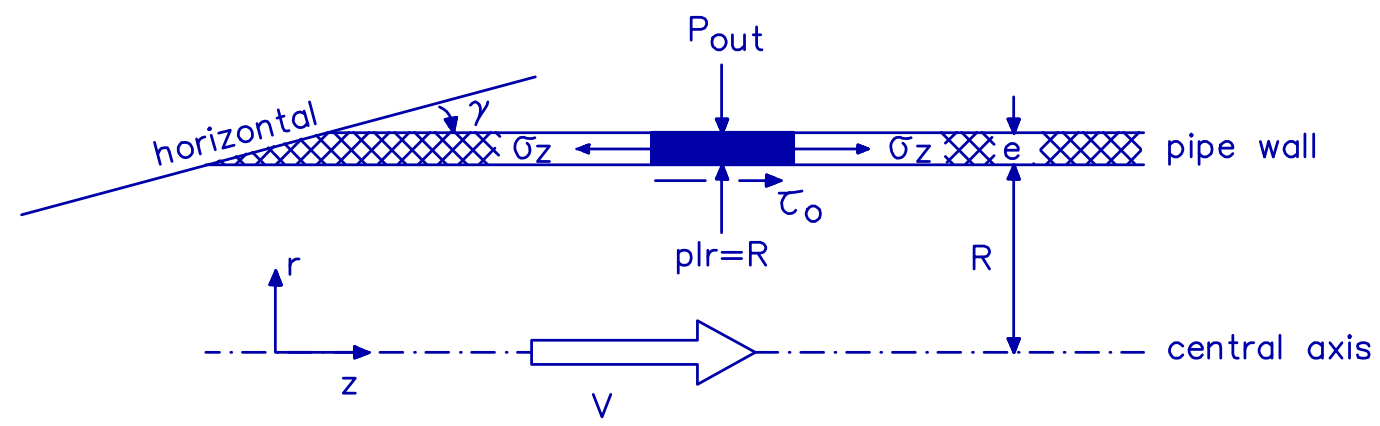

Fig. 1. Definition sketch, side view ( $z-r$ plane): stresses acting on pipe wall.

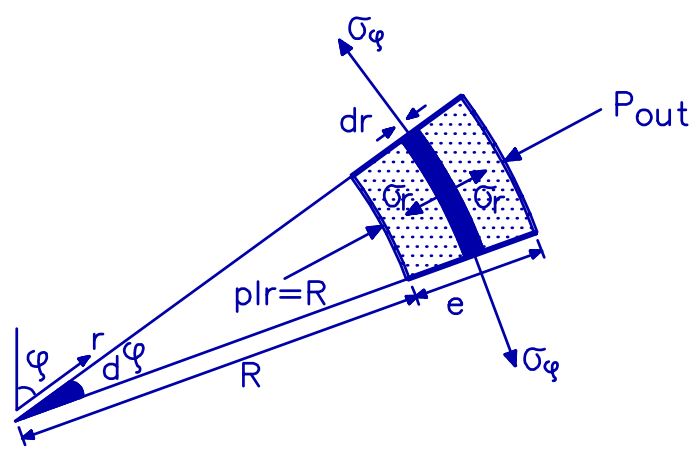

Fig. 2. Definition sketch, cross-sectional view ( $r$ - $\varphi$ plane): normal stresses acting on element of pipe wall.

To arrive at a one-dimensional formulation, the equations (8) and (9) are multiplied by $2 \pi r$, integrated with respect to $r$ from $R$ to $R+e$, and divided by $A_{t}=2 \pi\left(R+\frac{1}{2} e\right) e$ :

Equation of motion in axial direction (1D)

$$
\rho_{t} \frac{\partial \overline{\dot{u}_{z}}}{\partial t}=\frac{\partial \overline{\sigma_{z}}}{\partial z}
$$

Equation of motion in radial direction (1D)

$$
\rho_{t} \frac{\partial \overline{\dot{u}}_{r}}{\partial t}=\left.\frac{R+e}{\left(R+\frac{1}{2} e\right) e} \sigma_{r}\right|_{r=R+e}-\left.\frac{R}{\left(R+\frac{1}{2} e\right) e} \sigma_{r}\right|_{r=R}-\frac{1}{R+\frac{1}{2} e} \overline{\overline{\sigma_{\varphi}}}
$$

in which

$$
\overline{\dot{u}_{z}}=\frac{1}{2 \pi\left(R+\frac{1}{2} e\right) e} \int_{R}^{R+e} 2 \pi r \dot{u}_{z} \mathrm{~d} r \quad, \quad \overline{\dot{u}_{r}}=\frac{1}{2 \pi\left(R+\frac{1}{2} e\right) e} \int_{R}^{R+e} 2 \pi r \dot{u}_{r} \mathrm{~d} r
$$




$$
\overline{\sigma_{z}}=\frac{1}{2 \pi\left(R+\frac{1}{2} e\right) e} \int_{R}^{R+e} 2 \pi r \sigma_{z} \mathrm{~d} r \quad \text { and } \quad \overline{\overline{\sigma_{\varphi}}}=\frac{1}{e} \int_{R}^{R+e} \sigma_{\varphi} \mathrm{d} r
$$

are averaged values of $\dot{u}_{z}, \dot{u}_{r}, \sigma_{z}$ and $\sigma_{\varphi}$. A single bar denotes cross-sectional area averages, a double bar denotes line(ar) averages. The first integral in Eqs (12) is the "flow rate" in axial pipe movement, the third is the axial force in the pipe wall. The fourth integral is the hoop force in the pipe wall per unit area of (axial) wall surface.

The equations of motion relate axial velocities to axial stresses (Eq. 10), and radial velocities to radial and hoop stresses (Eq. 11). Stress-velocity relations will complete the mathematical model. Stress-strain relations are provided by the generalised Hooke's law. For a three-dimensional isotropic solid the normal strains $\varepsilon_{z}, \varepsilon_{\varphi}$ and $\varepsilon_{r}$ depend linearly on the normal stresses $\sigma_{z}, \sigma_{\varphi}$ and $\sigma_{r}$ :

Stress-strain relations $(3 D)$

$\varepsilon_{z}=\frac{1}{E}\left[\sigma_{z}-v\left(\sigma_{\varphi}+\sigma_{r}\right)\right], \quad \varepsilon_{\varphi}=\frac{1}{E}\left[\sigma_{\varphi}-v\left(\sigma_{z}+\sigma_{r}\right)\right], \quad \varepsilon_{r}=\frac{1}{E}\left[\sigma_{r}-v\left(\sigma_{z}+\sigma_{\varphi}\right)\right]$

which is equivalent with

$$
\begin{gathered}
\sigma_{z}=\frac{E}{(1+v)(1-2 v)}\left[(1-v) \varepsilon_{z}+v\left(\varepsilon_{\varphi}+\varepsilon_{r}\right)\right] \quad \text { or } \quad \sigma_{z}=\frac{E}{1-v^{2}}\left(\varepsilon_{z}+v \varepsilon_{\varphi}\right)+\frac{v}{1-v} \sigma_{r} \\
\sigma_{\varphi}=\frac{E}{1-v^{2}}\left(\varepsilon_{\varphi}+v \varepsilon_{z}\right)+\frac{v}{1-v} \sigma_{r} \quad \text { and } \quad \sigma_{r}=E \varepsilon_{r}+v\left(\sigma_{z}+\sigma_{\varphi}\right)
\end{gathered}
$$

where $E$ is Young's modulus of elasticity and $v$ is Poisson's ratio.

The strain-displacement $(\varepsilon-u)$ relations are:

$$
\varepsilon_{z}=\frac{\partial u_{z}}{\partial z}, \quad \varepsilon_{\varphi}=\frac{u_{r}}{r} \quad \text { and } \quad \varepsilon_{r}=\frac{\partial u_{r}}{\partial r}
$$

The axial stress $\sigma_{z}$ is expressed in displacements after substitution of Eq. (15) in the first of Eqs (14):

Axial stress-displacement relation $(2 D)$

$$
\sigma_{z}=\frac{E}{(1+v)(1-2 v)}\left[(1-v) \frac{\partial u_{z}}{\partial z}+v \frac{1}{r} \frac{\partial\left(r u_{r}\right)}{\partial r}\right]
$$

Differentiation with respect to $t$, multiplication by $2 \pi r$, integration with respect to $r$ from $R$ to $R+e$, and division by $A_{t}=2 \pi\left(R+\frac{1}{2} e\right) e$ leads to: 
Axial stress-velocity relation (1D)

$\frac{\partial \overline{\sigma_{z}}}{\partial t}=\frac{E}{(1+v)(1-2 v)}\left[(1-v) \frac{\partial \overline{\dot{u}}_{z}}{\partial z}+\left.v \frac{R+e}{\left(R+\frac{1}{2} e\right) e} \dot{u}_{r}\right|_{r=R+e}-\left.v \frac{R}{\left(R+\frac{1}{2} e\right) e} \dot{u}_{r}\right|_{r=R}\right]$

Equation (16) is basic for a full 2D analysis, but Eq. (17) is not appropriate for the present $1 \mathrm{D}$ investigation as will be explained in Section 3. An alternative equation is found by substituting the axial strain from Eq.

(15) in the first of Eqs (13), yielding

Axial stress-displacement relation (2D)

$$
\sigma_{z}=E \frac{\partial u_{z}}{\partial z}+v \sigma_{\varphi}+v \sigma_{r}
$$

and, applying the transformation between Eqs (16) and (17),

Axial stress-velocity relation (1D)

$$
\frac{\partial \overline{\sigma_{z}}}{\partial t}=E \frac{\partial \overline{\dot{u}_{z}}}{\partial z}+v \frac{\partial \overline{\sigma_{\varphi}}}{\partial t}+v \frac{\partial \overline{\sigma_{r}}}{\partial t}
$$

in which

$\overline{\sigma_{\varphi}}=\frac{1}{2 \pi\left(R+\frac{1}{2} e\right) e} \int_{R}^{R+e} 2 \pi r \sigma_{\varphi} \mathrm{d} r \quad$ and $\quad \overline{\sigma_{r}}=\frac{1}{2 \pi\left(R+\frac{1}{2} e\right) e} \int_{R}^{R+e} 2 \pi r \sigma_{r} \mathrm{~d} r$

\section{Liquid-pipe coupling}

The liquid and pipe equations are coupled by means of boundary conditions representing the contact between liquid and pipe wall on the interface at $r=R$. Outside the pipe a constant pressure, $P_{\text {out }}$, is assumed to exist. The interface conditions are

$$
\begin{array}{ll}
\left.\sigma_{r}\right|_{r=R}=-\left.p\right|_{r=R} & \left.\sigma_{r}\right|_{r=R+e}=-P_{\text {out }} \\
\left.\dot{u}_{r}\right|_{r=R}=\left.v_{r}\right|_{r=R} & \left.\dot{u}_{r}\right|_{r=R+e}=\left(v_{r}\right)_{\text {out }}
\end{array}
$$

where $\left(v_{r}\right)_{\text {out }}$ is the radial velocity of the external fluid. Buried pipes are not considered herein.

The dynamic conditions (21) give the fluid pressures acting on the pipe wall. The kinematic conditions (22) prescribe the adherence of solid and fluid. Except for its constant pressure, $P_{\text {out }}$, the fluid outside the pipe is not modelled, so that $\left(v_{r}\right)_{\text {out }}$ is not known. For this reason, and because the liquid inside the pipe will not be modelled two-dimensionally, the relations (22) do not provide suitable boundary conditions for pipe 
equation (17) [herein replaced by Eq. (19)]. Nevertheless, Eq. (17) may be useful in other applications. For example, with $\left.\dot{u}_{r}\right|_{r=R}=0$ and $\left.\dot{u}_{r}\right|_{r=R+e}=0$, Eq. (17) describes walls that are fixed in the radial direction [9, p. 64].

The conditions (21) are substituted in the equation of radial pipe motion (11). The condition (22), at $r=$ $R$, is substituted in the liquid equations (4) and (7). After the substitutions and a rearrangement of terms, eight basic equations remain for the eight variables $P, V, \sigma_{z}, \overline{\dot{u}_{z}}, \sigma_{\varphi}, u_{r}, \sigma_{r}$ and $\left.p\right|_{r=R}$. The eight equations stem from, successively, the relations (5), (4), (10), (19), (7), (11), and the second and third equations in (13) and (15). In summary:

Liquid, axial motion

$$
\begin{gathered}
\frac{\partial V}{\partial t}+\frac{1}{\rho_{f}} \frac{\partial P}{\partial z}=0 \\
\frac{\partial V}{\partial z}+\frac{1}{K} \frac{\partial P}{\partial t}+\left.\frac{2}{R} \dot{u}_{r}\right|_{r=R}=0
\end{gathered}
$$

Pipe, axial motion

$$
\begin{gathered}
\frac{\partial \overline{\dot{u}_{z}}}{\partial t}-\frac{1}{\rho_{t}} \frac{\partial \overline{\sigma_{z}}}{\partial z}=0 \\
\frac{\partial \overline{\dot{u}_{z}}}{\partial z}-\frac{1}{E} \frac{\partial \overline{\sigma_{z}}}{\partial t}+\frac{v}{E} \frac{\partial}{\partial t}\left(\overline{\sigma_{\varphi}}+\overline{\sigma_{r}}\right)=0
\end{gathered}
$$

Liquid, radial motion

$$
\left.p\right|_{r=R}=P-\left.\frac{1}{2} \rho_{f} R \frac{\partial \dot{u}_{r}}{\partial t}\right|_{r=R}
$$

Pipe, radial motion

$$
\begin{gathered}
\rho_{t} \frac{\partial \overline{\dot{u}_{r}}}{\partial t}=-\frac{R+e}{\left(R+\frac{1}{2} e\right) e} P_{\text {out }}+\left.\frac{R}{\left(R+\frac{1}{2} e\right) e} p\right|_{r=R}-\frac{1}{R+\frac{1}{2} e} \overline{\overline{\sigma_{\varphi}}} \\
u_{r}=\frac{r}{E}\left[\sigma_{\varphi}-v\left(\sigma_{z}+\sigma_{r}\right)\right] \\
\frac{\partial u_{r}}{\partial r}=\frac{1}{E}\left[\sigma_{r}-v\left(\sigma_{z}+\sigma_{\varphi}\right)\right]
\end{gathered}
$$

Substitution of expression (27) in equation (28) gives 
Equation of motion in radial direction

$$
\rho_{t}\left(R+\frac{1}{2} e\right) e \frac{\partial \overline{\dot{u}}_{r}}{\partial t}+\left.\frac{1}{2} \rho_{f} R^{2} \frac{\partial \dot{u}_{r}}{\partial t}\right|_{r=R}=R P-(R+e) P_{\text {out }}-e \overline{\overline{\sigma_{\varphi}}}
$$

Substitution of expression (29) in equation (24) gives:

Continuity equation

$$
\frac{\partial V}{\partial z}+\frac{1}{K} \frac{\partial P}{\partial t}+\frac{2}{E} \frac{\partial}{\partial t}\left[\left.\sigma_{\varphi}\right|_{r=R}-\left.v \sigma_{z}\right|_{r=R}-\left.v \sigma_{r}\right|_{r=R}\right]=0
$$

\section{FSI four-equation model for thick-walled pipes}

For long wavelengths (long compared to the radius of the pipe), accelerations in radial direction are negligible, so that the radial inertia terms in Eq. (31) can be left out $[8,10-16]$. Local effects near sharp wave fronts [17] are also neglected in the long-wave approximation. A quasi-static relation between the hoop stress and the internal pressure is the result,

$$
\overline{\overline{\sigma_{\varphi}}}=\frac{R}{e} P-\frac{R+e}{e} P_{o u t}
$$

Neglecting radial liquid inertia means that $p$ equals $P$; the pressure is uniform in each cross section of the pipe.

Equation (33) is confirmed in Appendix A, Eq. (54), where the quasi-static stress distribution in a pressurised ring is given. Furthermore, from the Eqs (57) and (53), it follows that,

$$
\begin{gathered}
\frac{\partial}{\partial t}\left(\overline{\sigma_{\varphi}}+\overline{\sigma_{r}}\right)=\frac{R}{e} \frac{1}{1+\frac{1}{2} \frac{e}{R}} \frac{\partial P}{\partial t} \\
\frac{\partial}{\partial t}\left(\left.\sigma_{\varphi}\right|_{r=R}\right)=\left(\frac{R}{e}+\frac{1+\frac{e}{R}}{2+\frac{e}{R}}\right) \frac{\partial P}{\partial t}
\end{gathered}
$$

Equation (34) is substituted in Eq. (26), and Eq. (35), together with $\left.\sigma_{r}\right|_{r=R}=-P$, is substituted in Eq. (32). The $r$-dependency of $\varepsilon_{z}$, and hence of $\sigma_{z}$, is considered to be small, which means that pipe crosssections remain almost plane when stretched axially. Then, the quantity $\left.\sigma_{z}\right|_{r=R}$ in Eq. (32) can be replaced by $\overline{\sigma_{z}}$. Note that $\sigma_{\varphi}+\sigma_{r}$, caused by an internal pressure $P$, is a function of $z$ and $t$ only [see Appendix A, Eq. (48) + Eq. (49)], so that according to Eq. (18) the hoop and radial stresses do not introduce $r$-dependency 
in $\sigma_{z}$. Four basic equations remain, Eqs (23), (32), (25), (26), for the four unknowns $P, V, \overline{\sigma_{z}}$ and $\overline{\dot{u}_{z}}$ :

Liquid, axial

$$
\begin{gathered}
\frac{\partial V}{\partial t}+\frac{1}{\rho_{f}} \frac{\partial P}{\partial z}=0 \\
\frac{\partial V}{\partial z}+\left[\frac{1}{K}+\frac{2}{E}\left(\frac{R}{e}+\frac{1+\frac{e}{R}}{2+\frac{e}{R}}+v\right)\right] \frac{\partial P}{\partial t}=\frac{2 v}{E} \frac{\partial \overline{\sigma_{z}}}{\partial t}
\end{gathered}
$$

Pipe, axial

$$
\begin{gathered}
\frac{\partial \overline{\dot{u}_{z}}}{\partial t}-\frac{1}{\rho_{t}} \frac{\partial \overline{\sigma_{z}}}{\partial z}=0 \\
\frac{\partial \overline{\dot{u}_{z}}}{\partial z}-\frac{1}{E} \frac{\partial \overline{\sigma_{z}}}{\partial t}=-\frac{v R}{E e} \frac{1}{1+\frac{1}{2} \frac{e}{R}} \frac{\partial P}{\partial t}
\end{gathered}
$$

This is the thick-wall FSI four-equation model. If the usual thin-wall assumption is made, in which $e / R$ is neglected with respect to unity, the four-equation model of Skalak [10] remains.

\section{Wave propagation speeds}

The four eigenvalues $\lambda$ of the hyperbolic system of partial differential equations $(36,37,38,39)$ represent the propagation speeds - in two directions - of axial waves in straight liquid-filled pipes. The magnitudes are obtained from the following bi-quadratic dispersion relation,

$$
\lambda^{4}-\gamma^{2} \lambda^{2}+c_{f}^{2} c_{t}^{2}=0
$$

with

$$
\gamma^{2}=\left(1+2 v^{2} \frac{\rho_{f}}{\rho_{t}} \frac{R}{e} \frac{1}{1+\frac{1}{2} \frac{e}{R}}\right) c_{f}^{2}+c_{t}^{2}
$$

This leads to modified (because of FSI) squared wave speeds

$$
\lambda_{1,2}^{2}=\frac{1}{2}\left[\gamma^{2}-\left(\gamma^{4}-4 c_{f}^{2} c_{t}^{2}\right)^{1 / 2}\right]
$$

and

$$
\lambda_{3,4}^{2}=\frac{1}{2}\left[\gamma^{2}+\left(\gamma^{4}-4 c_{f}^{2} c_{t}^{2}\right)^{1 / 2}\right]
$$


where $\lambda_{1}$ and $\lambda_{3}$ are positive, and $\lambda_{2}$ and $\lambda_{4}$ are negative. The constants

$$
c_{f}^{2}=\left\{\rho_{f}\left[\frac{1}{K}+\frac{2}{E}\left(\frac{R}{e}\left(1-\frac{v^{2}}{1+\frac{1}{2} \frac{e}{R}}\right)+\frac{1+\frac{e}{R}}{2+\frac{e}{R}}+v\right)\right]\right\}^{-1}
$$

and

$$
c_{t}^{2}=\frac{E}{\rho_{t}}
$$

are the squares of the classical pressure wave speed $[3,18,19]$ and the bar velocity [20, p. 493], respectively.

Taking the limit to zero of $e / R$, one obtains

$$
\lambda_{1,2}^{2}=\frac{E e}{2 \rho_{f} R\left(1-v^{2}\right)} \rightarrow 0 \quad \text { and } \quad \lambda_{3,4}^{2}=\frac{c_{t}^{2}}{1-v^{2}}
$$

which are the squares of the conventional pressure wave speed in flexible tubes filled with incompressible liquid and the plate velocity $[9$, p. 81], respectively. Taking the limit of $e / R$ to infinity, one obtains

$$
\lambda_{1,2}^{2}=\left\{\rho_{f}\left[\frac{1}{K}+\frac{2}{E}(1+v)\right]\right\}^{-1} \quad \text { and } \quad \lambda_{3,4}^{2}=c_{t}^{2}
$$

which are the squares of the conventional pressure wave speed in circular tunnels $[3,18,19]$ and the bar velocity, respectively.

It appears that the coupled pressure and stress waves travel without dispersion. Gromeka [21], Lamb [22], Skalak [10] and Lin and Morgan [11, 12] analysed this problem in the frequency domain, and Bürmann [23], Stuckenbruck et al. [24] and Leslie and Tijsseling [25] in the time domain. References [26] and [27] give extensive reviews of the subject.

\section{Exact solutions}

The FSI four-equation model $(36,37,38,39)$ can be solved exactly in the time $[28,29]$ and frequency $[30,31,32]$ domains. Some results are presented for the Dundee water-filled steel pipe $[33,34,35]$ with data given in Table 1, where the thickness/radius ratio $e / R=0.152$. The test pipe is $4.5 \mathrm{~m}$ long and has an inner radius of $26 \mathrm{~mm}$. A five metre long solid steel rod hits the pipe axially at one of its closed ends. The water in the pipe has a static pressure of typically $2 \mathrm{MPa}$ to prevent the occurrence of cavitation. Pressures, strains and structural velocities were measured at different positions along the pipe. 


\begin{tabular}{|c|c|}
\hline \multicolumn{2}{|c|}{ Steel pipe } \\
\hline length & $L=4.51 \mathrm{~m}$ \\
\hline inner radius & $R=26.01 \mathrm{~mm}$ \\
\hline wall thickness & $e=3.945 \mathrm{~mm}$ \\
\hline Young's modulus & $E=168 \mathrm{GPa}$ \\
\hline mass density & $\rho_{t}=7985 \mathrm{~kg} / \mathrm{m}^{3}$ \\
\hline Poisson's ratio & $v=0.29$ \\
\hline end mass at $z=0$ & $m_{0}=1.312 \mathrm{~kg}$ \\
\hline end mass at $z=L$ & $m_{L}=0.3258 \mathrm{~kg}$ \\
\hline wall area & $A_{t}=694 \mathrm{~mm}^{2}$ \\
\hline \multicolumn{2}{|c|}{ Water } \\
\hline bulk modulus & $K=2.14 \mathrm{GPa}$ \\
\hline mass density & $\rho_{f}=999 \mathrm{~kg} / \mathrm{m}^{3}$ \\
\hline flow area & $A_{f}=2125 \mathrm{~mm}^{2}$ \\
\hline
\end{tabular}

Table 1. Geometrical and material properties of Dundee test pipe, closed at both ends and freely suspended.

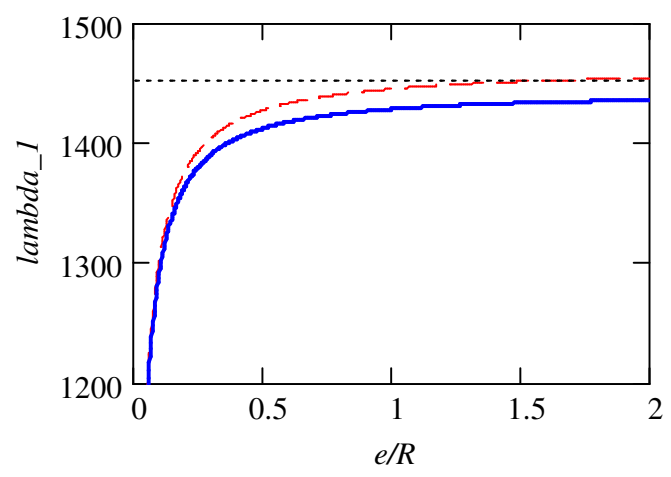

(a)

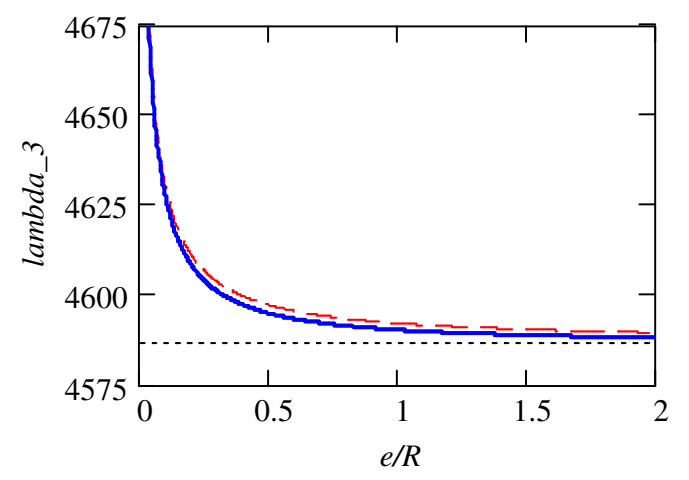

(b)

Fig. 3. Wave speeds (in m/s) (Eq. 42) as function of thickness/radius ratio for thick-wall theory (thick solid line), thin-wall theory (thin broken line) and limit values (Eq. 45) (dotted lines): (a) pressure wave speed $\lambda_{1}$, (b) axial stress wave speed $\lambda_{3}$. 


\begin{tabular}{|c|c|c|c|c|}
\hline $\begin{array}{c}\text { measurement } \\
{[31]}\end{array}$ & $\begin{array}{c}\text { thin-wall calculation } \\
\text { without } \\
\text { end masses }\end{array}$ & difference $(\%)$ & $\begin{array}{c}\text { thick-wall calculation } \\
\text { without } \\
\text { end masses }\end{array}$ & difference $(\%)$ \\
\hline 173 & 172 & -0.6 & 170 & -1.7 \\
\hline 289 & 286 & -1.0 & 284 & -1.7 \\
\hline 459 & 453 & -1.3 & 449 & -2.1 \\
\hline 485 & 493 & +1.6 & 491 & +1.2 \\
\hline 636 & 633 & -0.5 & 628 & -1.3 \\
\hline 750 & 741 & -1.2 & 735 & -2.0 \\
\hline 918 & 907 & -1.2 & 899 & -2.1 \\
\hline 968 & 980 & +1.2 & 976 & +1.0 \\
\hline
\end{tabular}

Table 2a. Natural frequencies (in $\mathrm{Hz}$ ) of axial vibration of closed water-filled steel pipe with free closed ends. Calculations without end masses.

\begin{tabular}{||c|c|c|c|c||}
\hline \multirow{2}{*}{$\begin{array}{c}\text { measurement } \\
{[31]}\end{array}$} & thin-wall calculation & & thick-wall calculation & difference $(\%)$ \\
\hline \hline 173 & end masses & difference $(\%)$ & end masses & -2.3 \\
\hline 289 & 171 & -1.2 & 169 & -2.1 \\
\hline 459 & 285 & -1.4 & 283 & -2.2 \\
\hline 485 & 453 & -1.3 & 449 & -3.1 \\
\hline 636 & 471 & -2.9 & 470 & -2.4 \\
\hline 750 & 626 & -1.6 & 621 & -2.1 \\
\hline 918 & 740 & -1.3 & 734 & -2.1 \\
\hline 968 & 906 & -1.3 & 899 & -2.6 \\
\hline
\end{tabular}

Table 2b. Natural frequencies (in $\mathrm{Hz}$ ) of axial vibration of closed water-filled steel pipe with free closed ends. Calculations with lumped end masses. 
The wave speeds calculated from Eqs (42) are shown in Fig. 3 as a function of $e / R$. The pressure wave speed $\lambda_{1}$ in Fig. 3(a) increases, from zero at $e / R=0$ to the tunnel velocity of $1452 \mathrm{~m} / \mathrm{s}$ (Eq. 45) at $e / R=\infty$, because the pipe hoop stiffness increases with $e / R$. The stress wave speed $\lambda_{3}$ in Fig. 3(b) decreases, from the plate velocity of $4793 \mathrm{~m} / \mathrm{s}($ Eq. 44$)$ at $e / R=0$ to the bar velocity of $4587 \mathrm{~m} / \mathrm{s}(\mathrm{Eq} .45)$ at $e / R=\infty$, because the wall thickness increases. Thick-wall theory predicts lower pressure wave speeds, because of additional mass of the pipe wall, and higher stress wave speeds, because of a stiffer wall. The differences between thick-wall theory (thick solid lines) and thin-wall theory (where $e / R$ terms are neglected with respect to unity) (thin broken lines) are less than $1.5 \%$ for the pressure wave speed and less than $0.05 \%$ for the stress wave speed, over the whole $e / R$ range. These small differences return in the calculated natural frequencies listed in Table 2, where the lower pressure wave speeds predicted by thick-wall theory lead to lower frequencies, even for the "structural" frequencies measured at $485 \mathrm{~Hz}\left[\approx \lambda_{3} /(2 L)\right]$ and $968 \mathrm{~Hz}\left[\approx \lambda_{3} / L\right]$. Table 2 lists calculated results obtained without [Table 2(a)] and with [Table 2(b)] taking into account the lumped masses closing the pipe. The thick-wall results in the third column are furthest away from the measured data in the first column, but the last column of Table 2(b) shows the most systematic deviation from the experiment in the sense that all calculated frequencies are $2 \%$ to $3 \%$ lower than the measured ones. In this respect one could remark that the statically measured modulus of elasticity of $168 \pm 5 \mathrm{GPa}[33$, p. 28] is rather low for stainless steel.

The time-domain results, obtained without including the lumped end masses, and shown in Fig. 4, confirm the observations above. Thick-wall theory predicts a slightly slower transient, which slowly departs from the thin-wall result, in phase and amplitude, as time increases. The inclusion of the end masses, of friction and damping mechanisms [36], and of a non-instantaneous excitation, will give more realistic computational results, but exact solutions like those in Fig. 4 cannot be obtained.

Wall thickness may be of importance in the determination of fluid pressures from (measured) hoop strains. The hoop strain $\varepsilon_{\varphi}$ is calculated at the outer radius $r=R+e$, because strain gauges are normally glued to the external surface of the pipe. Substituting $\left.\sigma_{\phi}\right|_{r=R+e}[$ from Eqs (48), (51) and (52)] and $\left.\sigma_{r}\right|_{r=R+e}=-P_{\text {out }}$ into the second of Eqs (13), and ignoring the (usually) unknown axial stresses $\sigma_{z}$, one obtains:

$$
\varepsilon_{\varphi}=\frac{1}{E}\left(\frac{R}{e} \frac{1}{1+\frac{1}{2} \frac{e}{R}}\left(P-P_{\text {out }}\right)-(1-v) P_{\text {out }}\right)
$$


where $P$ and $P_{\text {out }}$ are the internal and external pressure, respectively. The thick-wall equation (46) had to be used to accurately predict the hoop strains in the Dundee test pipe [33, p. 44, p. 47] .The relative pressure is derived from the hoop strain by

$$
P-P_{\text {out }}=E \frac{e}{R}\left(1+\frac{1}{2} \frac{e}{R}\right)\left(\varepsilon_{\varphi}-\varepsilon_{\varphi, \text { out }}\right)
$$

where $\varepsilon_{\varphi, \text { out }}=-(1-v) P_{\text {out }} / E$ is the hoop strain when $P=P_{\text {out }}$.

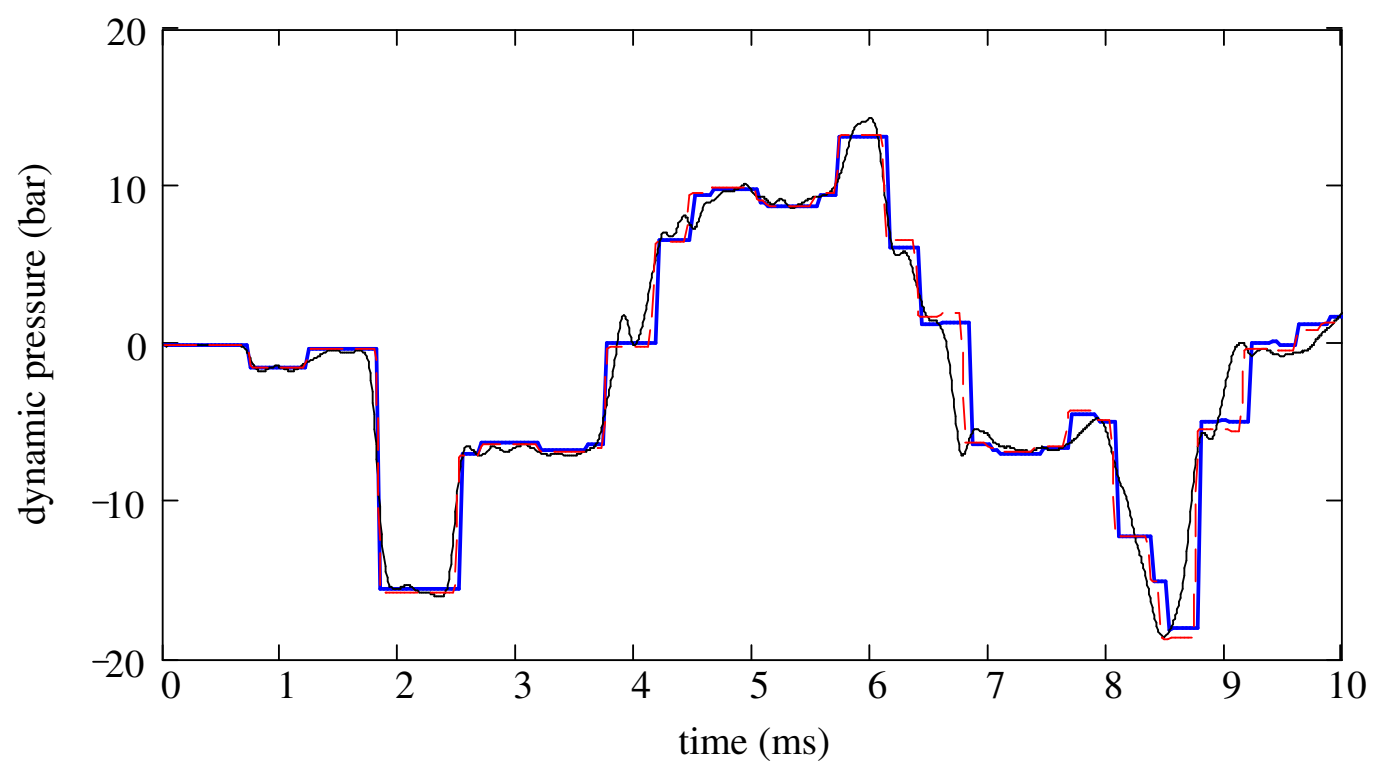

Fig. 4. Dynamic pressure in Dundee single pipe experiment (pressure $3.376 \mathrm{~m}$ away from the impact end). Thick solid line: thick-wall theory; thin broken line: thin-wall theory; thin solid line: experiment.

\section{Conclusion}

A rigorous derivation of one-dimensional equations describing fluid-structure interaction mechanisms in the axial/radial vibration of liquid-filled pipes has been presented, thereby taking the thickness of the pipe wall into account through the averaging of hoop and radial stresses. FSI coupled wave speeds have been formulated and investigated. It has been shown, for one example concerning a water-filled steel pipe, that the thin-wall assumption is valid for fairly thick pipes. Liquid frictional and structural damping effects are usually small and have been neglected herein, but these could be included in the analysis $[4,5,31,36]$. 


\section{Appendix A}

\section{Two-dimensional stress distribution in a pressurised ring}

In the present investigation the radial inertia forces in both liquid and pipe wall are neglected. For the liquid this means that the pressure is uniform in each pipe cross-section, whereas for the pipe a quasi-static stress distribution across the thickness of the pipe wall is assumed. This particular stress distribution, given in [20, pp. 68-71] and attributed to Lamé [37], is integrated here over the pipe wall cross-section.

Consider the two-dimensional axially symmetric stress problem of a circular ring subjected to an internal pressure $P$ and an external pressure $P_{\text {out }}$. The ring, shown in Fig. 5, has inner radius $R$ and thickness $e$. The hoop and radial stresses have the form

$$
\begin{gathered}
\sigma_{\varphi}=-\frac{A}{r^{2}}+2 C \\
\sigma_{r}=\frac{A}{r^{2}}+2 C
\end{gathered}
$$

where the constants of integration, $A$ and $C$, are determined from the boundary conditions

$$
\left.\sigma_{r}\right|_{r=R}=-P(z, t) \quad \text { and }\left.\quad \sigma_{r}\right|_{r=R+e}=-P_{\text {out }}
$$

It follows that

$$
\begin{aligned}
& A=\frac{R^{2}(R+e)^{2}\left(P_{\text {out }}-P\right)}{2\left(R+\frac{1}{2} e\right) e} \\
& 2 C=\frac{R^{2} P-(R+e)^{2} P_{\text {out }}}{2\left(R+\frac{1}{2} e\right) e}
\end{aligned}
$$

The stress solutions $\sigma_{\varphi}$ and $\sigma_{r}$ are independent of Poisson's ratio $v$, so that they are valid for both (axial) plane stress and (axial) plane strain situations. The sum $\sigma_{\varphi}+\sigma_{r}$ is equal to $4 C$ and therefore independent of $r$.

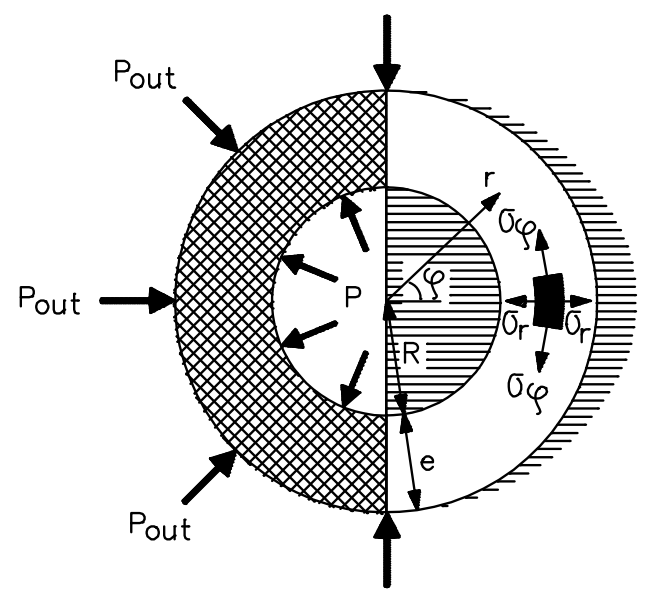

Fig. 5. Definition sketch of stress distribution in a pressurised ring. 
The value of $\sigma_{\varphi}$ at $r=R$ follows from (48), (51) and (52),

$$
\left.\sigma_{\varphi}\right|_{r=R}=-\frac{A}{R^{2}}+2 C=\frac{R}{e} P-\frac{R+e}{e} P_{\text {out }}+\frac{1+\frac{e}{R}}{2+\frac{e}{R}}\left(P-P_{\text {out }}\right)
$$

The averaged value of $\sigma_{\varphi}$, defined in Eqs (12) and calculated with (48), (51) and (52), is

$$
\overline{\overline{\sigma_{\varphi}}}=\frac{1}{e} \int_{R}^{R+e} \sigma_{\varphi} \mathrm{d} r=-\frac{A}{R(R+e)}+2 C=\frac{R}{e} P-\frac{R+e}{e} P_{\text {out }}
$$

The other averaged value of $\sigma_{\varphi}$, defined in Eqs (20) and calculated with (48), (51) and (52), is

$$
\begin{gathered}
\overline{\sigma_{\varphi}}=\frac{1}{2 \pi\left(R+\frac{1}{2} e\right) e} \int_{R}^{R+e} 2 \pi r \sigma_{\varphi} \mathrm{d} r=-\frac{\ln \left(1+\frac{e}{R}\right)}{\left(R+\frac{1}{2} e\right) e} A+2 C= \\
=\left[\frac{R^{2}}{2 e^{2}} \frac{(R+e)^{2} \ln \left(1+\frac{e}{R}\right)+\left(R+\frac{1}{2} e\right) e}{\left(R+\frac{1}{2} e\right)^{2}}\right] P-\left[\frac{R^{2}}{2 e^{2}} \frac{(R+e)^{2} \ln \left(1+\frac{e}{R}\right)+\left(1+\frac{e}{R}\right)^{2}\left(R+\frac{1}{2} e\right) e}{\left(R+\frac{1}{2} e\right)^{2}}\right] P_{\text {out }}
\end{gathered}
$$

The averaged value of $\sigma_{r}$, defined in Eqs (20) and calculated with (49), (51) and (52), is

$$
\begin{gathered}
\overline{\sigma_{r}}=\frac{1}{2 \pi\left(R+\frac{1}{2} e\right) e} \int_{R}^{R+e} 2 \pi r \sigma_{r} \mathrm{~d} r=\frac{\ln \left(1+\frac{e}{R}\right)}{\left(R+\frac{1}{2} e\right) e} A+2 C= \\
=\left[-\frac{R^{2}}{2 e^{2}} \frac{(R+e)^{2} \ln \left(1+\frac{e}{R}\right)-\left(R+\frac{1}{2} e\right) e}{\left(R+\frac{1}{2} e\right)^{2}}\right] P+\left[\frac{R^{2}}{2 e^{2}} \frac{(R+e)^{2} \ln \left(1+\frac{e}{R}\right)-\left(1+\frac{e}{R}\right)^{2}\left(R+\frac{1}{2} e\right) e}{\left(R+\frac{1}{2} e\right)^{2}}\right] P_{\text {out }}
\end{gathered}
$$

The sum of the averaged values of $\sigma_{\varphi}$ and $\sigma_{r}$, defined in Eqs (20), and calculated with (48), (49), (51) and (52), is

$$
\overline{\sigma_{\varphi}}+\overline{\sigma_{r}}=\frac{1}{2 \pi\left(R+\frac{1}{2} e\right) e} \int_{R}^{R+e} 2 \pi r\left(\sigma_{\varphi}+\sigma_{r}\right) \mathrm{d} r=4 C=\frac{R}{e} \frac{1}{1+\frac{1}{2} \frac{e}{R}} P-\frac{R}{e} \frac{\left(1+\frac{e}{R}\right)^{2}}{1+\frac{1}{2} \frac{e}{R}} P_{\text {out }}
$$

For thin-walled pipes, $e<<R$, the approximation $\ln (1+e / R) \approx e / R$ is made. Terms of the order of $e / R$, and higher, are neglected with respect to unity. The averaged values of $\sigma_{\varphi}$ and $\sigma_{r}$, given by the expressions (54), (55) and (56), then become

$$
\begin{gathered}
\overline{\sigma_{\varphi}}=\overline{\overline{\sigma_{\varphi}}}=\frac{R}{e}\left(P-P_{\text {out }}\right) \\
\overline{\sigma_{r}}=-\frac{3}{4} P-\frac{1}{4} P_{\text {out }}
\end{gathered}
$$




\section{References}

[1] Koetzier H, Kruisbrink ACH, Lavooij CSW. Dynamic behaviour of large non-return valves. In: Papworth M, editor. Proceedings of the 5th International Conference on Pressure Surges, 237-243. Cranfield, UK: BHRA, 1986.

[2] Bergant A, Simpson AR, Tijsseling AS. Water hammer with column separation: A historical review. Journal of Fluids and Structures 2006; 22(2): 135-171.

[3] Wylie EB, Streeter VL. Fluid transients in systems. Englewood Cliffs, New Jersey, USA: Prentice Hall, 1993.

[4] Tijsseling AS. Fluid-structure interaction in case of waterhammer with cavitation. Ph.D. Thesis, Delft University of Technology, Faculty of Civil Engineering, Communications on Hydraulic and Geotechnical Engineering, Report No. 93-6, ISSN 0169-6548, Delft, The Netherlands, 1993; www.darenet.nl/en/page/language.view/search.page.

[5] Zhang YL, Vairavamoorthy K. Analysis of transient flow in pipelines with fluid-structure interaction using method of lines. International Journal for Numerical Methods in Engineering 2005; 63(10) 14461460.

[6] Walker JS, Phillips JW. Pulse propagation in fluid-filled tubes. ASME Journal of Applied Mechanics 1977; 44(March): 31-35.

[7] Kellner A, Schönfelder C. The effect of fluid/structure-interaction on pressure pulse loads on pipes. 3R international 1982; 21(8): 443-449 (in German).

[8] Barez F, Goldsmith W, Sackman JL. Longitudinal waves in liquid-filled tubes - I. Theory, II. Experiments. International Journal of Mechanical Sciences 1979; 21(4): 213-236.

[9] Kolsky H. Stress waves in solids. New York, USA: Dover Publications, 1963.

[10] Skalak, R. An extension of the theory of water hammer. Transactions of the ASME 1956; 78(1): 105-116.

[11] Lin TC, Morgan GW. A study of axisymmetric vibrations of cylindrical shells as affected by rotatory inertia and transverse shear. ASME Journal of Applied Mechanics 1956; 23(June): 255-261.

[12] Lin TC, Morgan GW. Wave propagation through fluid contained in a cylindrical, elastic shell. Journal of the Acoustical Society of America 1956; 28(6): 1165-1176.

[13] Meißner E. Influence of radial inertia of a pipe wall on non-stationary flow phenomena in pressurized 
conduits. Technische Universität München, Hydraulik und Gewässerkunde, Mitteilungen, Heft 25, 4362, Munich, Germany, 1978 (in German).

[14] Schwarz W. Waterhammer calculations taking into account the radial and longitudinal displacements of the pipe wall. Ph.D. Thesis, Universität Stuttgart, Institut für Wasserbau, Mitteilungen, Heft 43, Stuttgart, Germany, 1978 (in German).

[15] Bürmann W. Longitudinal motion of pipelines laid in the open due to water hammer. 3R international 1980; 19(1-2): $84-91$ (in German).

[16] Kuiken GDC. Amplification of pressure fluctuations due to fluid-structure interaction. Journal of Fluids and Structures 1988; 2(5): 425-435.

[17] Tijsseling AS, Lambert MF, Simpson AR, Stephens ML, Vítkovský JP, Bergant A. Wave front dispersion due to fluid-structure interaction in long liquid-filled pipelines. In: Kurokawa J, editor. Proceedings of the 23rd IAHR Symposium on Hydraulic Machinery and Systems, Paper 143, Yokohama, Japan, October 2006.

[18] Halliwell AR. Velocity of a water-hammer wave in an elastic pipe. ASCE Journal of the Hydraulics Division 1963; 89(4): 1-21 (discussed by VL Streeter in 89(6): 295-296).

[19] Thorley ARD. Fluid transients in pipeline systems: a guide to the control and suppression of fluid transients in liquids in closed conduits (2nd edition). London: Professional Engineering Publishing, 2004.

[20] Timoshenko SP, Goodier JN. Theory of elasticity (3rd edition). Singapore: McGraw-Hill, 1970.

[21] Gromeka IS. On the velocity of propagation of wave-like motion of fluids in elastic tubes. PhysicalMathematical Section of the Scientific Society of the Imperial University of Kazan, Kazan, Russia, 1883: 1-19 (in Russian).

[22] Lamb H. On the velocity of sound in a tube, as affected by the elasticity of the walls. Memoirs of the Manchester Literary and Philosophical Society, Manchester, UK, 1898; 42(9): 1-16.

[23] Bürmann W. Water hammer in coaxial pipe systems. ASCE Journal of the Hydraulics Division 1975; 101(6): 699-715 [Errata in 101(12): 1554].

[24] Stuckenbruck S, Wiggert DC, Otwell RS. The influence of pipe motion on acoustic wave propagation. ASME Journal of Fluids Engineering 1985; 107(4): 518-522.

[25] Leslie DJ, Tijsseling AS. Wave speeds and phase velocities in liquid-filled pipes. In: Půlpitel L, editor. Proceedings of the 9th International Meeting of the IAHR Work Group on the Behaviour of Hydraulic 
Machinery under Steady Oscillatory Conditions, Paper E1, Brno, Czech Republic, September 1999.

[26] Tijsseling AS. Fluid-structure interaction in liquid-filled pipe systems: a review. Journal of Fluids and Structures 1996; 10(2): 109-146.

[27] Wiggert DC, Tijsseling AS. Fluid transients and fluid-structure interaction in flexible liquid-filled piping. ASME Applied Mechanics Reviews 2001; 54(5): 455-481.

[28] Li QS, Yang K, Zhang L. Analytical solution for fluid-structure interaction in liquid-filled pipes subjected to impact-induced water hammer. ASCE Journal of Engineering Mechanics 2003; 129(12): 1408-1417.

[29] Tijsseling AS. Exact solution of linear hyperbolic four-equation systems in axial liquid-pipe vibration. Journal of Fluids and Structures 2003; 18(2): 179-196.

[30] de Jong CAF. Analysis of pulsations and vibrations in fluid-filled pipe systems. Ph.D. Thesis, Eindhoven University of Technology, Department of Mechanical Engineering, Eindhoven, The Netherlands, 1994, Errata: April 2000; www.darenet.nl/en/page/language.view/search.page.

[31] Zhang L, Tijsseling AS, Vardy AE. FSI analysis of liquid-filled pipes. Journal of Sound and Vibration 1999; 224(1): 69-99.

[32] Brown FT, Tentarelli SC. Dynamic behavior of complex fluid-filled tubing systems — Part 1: Tubing analysis. Journal of Dynamic Systems, Measurement, and Control 2001; 123: 71-77.

[33] Fan D. Fluid-structure interactions in internal flows. Ph.D. Thesis, The University of Dundee, Department of Civil Engineering, Dundee, UK, 1989.

[34] Vardy AE, Fan D. Flexural waves in a closed tube. In: Thorley ARD, editor. Proceedings of the 6th International Conference on Pressure Surges, 43-57. Cranfield, UK: BHRA, 1989.

[35] Tijsseling AS, Vardy AE. Twenty years of FSI experiments in Dundee. In: Bathe KJ, editor. Proceedings of the Third M.I.T. Conference on Computational Fluid and Solid Mechanics, 10141017. Oxford, UK: Elsevier, 2005.

[36] Leslie DJ, Tijsseling AS. A review of modelling damping mechanisms in coupled liquid-pipe vibrations. In: Liou JCP, editor. Symposium S-290 Water Hammer, Proceedings of the 3rd ASME \& JSME Joint Fluids Engineering Conference, ASME - FED 248, Paper FEDSM99-6878, New York, USA: ASME, 1999.

[37] Lamé G. Lessons in the theory of elasticity. Paris: Gauthier-Villars, 1852 (in French). 